\section{Early symptoms of bone and soft tissue sarcomas: could they be diagnosed earlier?}

Mike Maguire

doi: 10.1308/003588412X13373405386213

\section{CORRESPONDENCE TO}

Mike Maguire, E: mrmmaguire@hotmail.com

\section{COMMENT ON}

\section{George A, Grimer R}

Early symptoms of bone and soft tissue sarcomas: could they be diagnosed earlier? Ann R Coll Surg Eng/ 2012; 94: 261-266 doi: 10.1308/003588412X13171221590016

The thrust of the above article seems to be that GP 'awareness and referral of sarcomas remain poor' to specialist centres and it suggests 'amendments to current guidelines and clearer referral pathways' and 'robust education strategies' for GPs. To put this in context of primary care: given that there are 2,700 cases per year in the UK in a population of 50 million adults, an average GP list size of 1,800 patients and $90 \%$ of cases presenting to the GP, a rough estimate is that a GP would expect to see a sarcoma every 11.4 years or 2-3 cases in his or her working life. I also note that the peak incidence is the eighth decade for soft tissue sarcomas and seventh decade for bone sarcomas.

Regarding the methodology, it is not clear from what origin the information came to complete 'a semi-structured proforma', which included the essential information about when the patient presented to the GP initially and what the initial symptoms were. It is hoped that this was gleaned from the contemporaneous GP notes accessed through the GPs themselves, which I feel would carry some significance. However, information gathered purely from patient recollections in hindsight following the diagnosis and probable (painful) treatment would have low accuracy.

Despite the apparent delay in referral to a specialist centre there is no discussion as to why this is, apart from a comment about 'lack of awareness'. This study has no evidence to support such a statement. The authors could have discussed the limitations of the diagnostic criteria by which individual symptoms are likely to have very low specificity (with the exception of a mass) in patients in their 60 s and 70 s who commonly have painful arthritides. Diagnosis is not clinched by specialist centres but by accurately reported imaging, the access for which is variable for GPs.

Once a diagnosis is made the pathways are very clear through the two-week wait system. Do the authors feel that the explicit pressure on GPs to refer less to specialist centres through financial penalties if GPs refer too much might be a contributing factor? I am unsure whether the authors are aware of the national cancer strategy for England (January 2011), which incorporates health promotion, encouraging earlier presentation, more screening and better access to GPs for diagnostic tests, and the use of cancer risk scores (from the QResearch database or the Risk Assessment Tool), which identified symptoms or coded variables in primary care to improve cancer outcomes.

\section{Not just a vampire repellent: the adverse effects of garlic supplements in surgery}

A Lawn, P Sains

Western Sussex Hospitals NHS Trust

doi: 10.1308/003588412X13373405385098

CORRESPONDENCE TO

Anneliese Lawn, alawn@doctors.org.uk

Apart from its culinary and mythical uses, garlic also has beneficial medicinal properties including antimicrobial, antiviral, antifungal and anti-cancer effects; and preventing heart disease by aiding the control of hypertension. ${ }^{1}$ Nevertheless, our experience has noted a deleterious effect from supplementary garlic consumption that has affected our clinical practice in colorectal surgery.

Garlic is known to be a blood thinner due its anti-platelet properties. Ajoene, a sulphur containing derivative of garlic, irreversibly inhibits platelet aggregation, ${ }^{2}$, potentiating anticoagulants such as aspirin, warfarin, dipyrimadole and clopidogrel. ${ }^{5,4}$ The composition of the garlic supplement affects its potency. Oil macerates have the highest content of ajoene and therefore exert the most potent effect. ${ }^{5}$

We performed elective high anterior resections for malignancy on two patients with no previous medical history, normal clotting profiles, platelet counts and not on anticoagulants or antiplatelets. Both laparoscopic cases converted to open due to the presence of generalised capillary ooze. Due to poor haemostasis, the risk of anastomotic leakage was high. Defunctioning ileostomies were formed despite original plans to perform a primary anastomosis. Retrospectively the patients admitted to self-medicating with garlic remedies. Recent recommendations from anaesthetic journals suggest that garlic supplements should be stopped seven days prior to operative intervention. ${ }^{6}$

\section{References}

1. Kaschula CH, Hunter R, Parker MI. Garlic-derived anticancer agents: structure and biological activity of ajoene. Biofactors 2010; 36: 78-85.

2. Srivastava KC, Tyagi OD. Effects of a garlic-derived principle (ajoene) on aggregation and arachidonic acid metabolism in human blood platelets. Prostaglandins Leukot Essent Fatty Acids 1993; 49: 587-95.

3. Heck AM, DeWitt BA, Lukes AL. Potential interactions between alternative therapies and warfarin. Am J Health Syst Pharm 2000; 57: 1,221-27; quiz 1,228-30.

4. Izzo AA, Di Carlo G, Borrelli F, Ernst E. Cardiovascular pharmacotherapy and herbal medicines: the risk of drug interaction. Int J Cardiol 2005; 98: 1-14.

5. Lawson LD, Ransom DK, Hughes BG. Inhibition of whole blood plateletaggregation by compounds in garlic clove extracts and commercial garlic products. Thromb Res 1992; 65: 141-56.

6. Wong A, Townley S. Herbal Medicines and Anaesthesia. Continuing Education in Anaesthetics, Critical Care and Pain 2010; 11: 14-17. doi: 10.1093/ bjaceaccp/mkq046. 